\section{MS5-P59 Structural and enzymatic kinetic studies of the chloroplast gamma-ketol reductase from Arabidopsis thaliana}

Sarah Mas y mas ${ }^{1}$, Curien Gilles ${ }^{2}$, Giustini Cécile ${ }^{2}$, Ferrer Jean-Luc $^{1}$, Rolland Norbert ${ }^{2}$, Cobessi David ${ }^{1}$

1. Institut de Biologie Structurale, Université Grenoble Alpes, CNRS, CEA, 71 Avenue des Martyrs, 38044 Grenoble, France

2. Laboratoire de Physiologie Cellulaire et Végétale, CNRS, Université Grenoble Alpes, CEA, INRA, 17 Rue des Martyrs, 38054 Grenoble, France

email: sarah.mas-y-mas@ibs.fr

In the course of the production of the signaling hormone jasmonate in chloroplast, gamma-ketol is released in the 13-LOX pathway. This highly reactive compound can damage lipids, proteins and DNA. Therefore it must be reduced. The chloroplast envelope Quinone OxidoReductase Homolog (ceQORH) from Arabidopsis thatiana represents 1 to $2 \%$ of the protein envelope. It binds NADPH, lacks a classical $N$-terminal and cleavable chloroplast transit peptide. It is transported through the chloroplast envelope by an unknown alternative pathway without cleavage of its internal chloroplast targeting sequence [1], [2]. We showed that this enzyme binds NADPH and reduces gamma-ketol [3]. Therefore, it has been renamed gamma-ketol reductase. It is inhibited by the ketodiens 13-KOTE and 13-KODE [3]. Using X-ray crystallography and analytical ultracentrifugation, we showed that the gamma-ketol reductase displays several oligomerization states [4]: the apo enzyme is a dimer, holoenzyme is a monomer and the enzyme bound to inhibitor is a tetramer. Structure analysis also revealed that the ligand binding site is large and hydrophobic allowing the enzyme to bind a broad range of ligands with a high affinity for gamma-ketol. By contrast with the other quinone oxydoreductases which dimerize by making a 12 stranded beta-sheet, the gamma-ketol reductase dimerizes through interaction of 2 alpha-helices from the Rossmann fold. These structural characteristics and the enzyme properties make ceQORH a new class distinct from the quinone oxydoreductases.

[1] Miras et al.,(2002) . J. Biol. Chem. 227 : 47770-47778

[2] Miras et al., (2007). J. Biol. Chem. $282: 29482-29492$

[3] Curien et al.,(2015) (submitted)

[4] Mas y Mas et al., (2015). Acta Cryst F. $71: 455-458$

Keywords: ceQORH, Chloroplast, gamma-ketol, X-ray crystallograph, analytical ultracentrifugation, oligomerization states

\section{MS5-P60 Characterization of enzymes} involved in biosynthesis and modification of sialic acids and their derivatives

\author{
Ronny Helland ${ }^{1}$
}

1. The Arctic University of Norway, Norway

email: ronny.helland@uit.no

Sialic acids play important roles in biological, pathological, and immunological processes, but they may also be building blocks for development of new drugs, neutraceuticals or high-value components in cosmetics. Sugar-modifying enzymes can therefore be utilized in many different areas of molecular biology and medicine, and a combination of chemical and enzymatic synthesis has the potential to produce new saccharides with useful properties.

The project aims to identify and characterize sugar-modifying enzymes from marine cold adapted species in order to improve the fundamental understanding of their role in bacterial pathogenesis and to identify targets for pharmaceutical and commercial exploitation. The project further aims at identifying enzymes which can be utilized in biomass conversion, i.e. from chitin or cellulose to high value products in medicine, cosmetics and food industry.

$\mathrm{N}$-acetylneuramininate lyase (Nal), catalyzing the reversible cleavage of $\mathrm{N}$-acetylneuraminic acid (Neu5Ac, also often called sialic acid) to N-acetylmannosamine (ManNAc) and pyruvate, is used as model system for exploring the catalytic mechanism and substrate/product modulation.

Keywords: sialic acids, carbohydrate chemistry, protein crystallography 